\title{
Instrumentation of a self-luminous fixation annulus
}

\author{
ALEEZA CERF-BEARE and LYNN LEACH \\ University of Maryland Baltimore County, Baltimore, Maryland 21228
}

\begin{abstract}
A simple and inexpensive way of constructing a fixation annulus is described. Such a device is important for experiments in which a fixation spot in the test field area is undesirable or when subjects' experimental tasks preclude their manipulation of a fixation dot brightness control. A luminous compound is inserted into transparent plastic tubing which can be shaped and inserted into a groove milled to fit the tube's diameter and shape. Annulus luminance is in the scotopic range. Repeated measurement showed that there was no brightness loss over time. Ways of further attenuating brightness are discussed.
\end{abstract}

The implementation of a luminous fixation spot in the stimulus test field may be impracticable for some forms of instrumentation or, in fact, contraindicated for vision experiments in which such a spot is undesirable. The rheostat-controlled "grain of wheat" bulb used in experiments in which dark adaptation is to be maintained requires the subject to make rheostat adjustments on a more or less continuous basis. The method which introduces a shiny bead with light reflected off it (Riggs, 1965) presupposes an independent entry of a reflecting beam into the optical pathway. Imposing a cross-hair reticle or a black fixation dot onto the test field (Boynton, 1966) presupposes a background luminance against which these are viewed. Finally, in experiments in which stimulus patterns, stimulus areas, or stimulus orientations are varied, the inclusion of a fixation dot or pattern in the stimulus configuration may be undesirable.

A set of experiments conducted in our laboratory involve virtually all of the problems inherent in fixation-spot instrumentation. Our stimuli vary in area, configuration, and orientation; and the addition of a tixation pattern anywhere within the test field would interfere with the stimulus, and thus potentially with the response. While central fixation is required for each exposure, subjects are maintained in the dark-adapted state between exposures. The stimuli are viewed through a $2-\mathrm{mm}$ artificial pupil, so that any tixation pattern also enables the subject to locate the test field before each exposure. Subjects are required to press one of two, or both, switches to indicate their responses to each exposure, which makes it virtually impossible to include any additional manipulandum, such as a rheostat. The stimuli are mounted onto a centering rotating mount to which the viewing tube is attached. Particular stimulus configurations can thus be rotated to present different stimulus orientations. This arrangement precludes the entry of an external beam for purposes of reflecting off a bead or set of beads, or the introduction of a fiber-optics pattern, without moditications to the equipment, especially without problems associated with light leakage into the visual pathway.

Since the entire field had to remain unencumbered, we decided on a fixation ring to surround the test field as an annulus. In this way, with appropriate instructions, central fixation of the stimulus is facilitated with the provision of peripheral orientation (Cornsweet. 1970). The material suitable for this purpose is Yellow Illuminance Compound 52-951 (HR Superior). It is nontoxic (used to paint numerals and dials on watch faces) and obtainable from jewelry supply houses. To preserve the requisite geometrical arrangement (Boynton, 1966), we experimented with painting dots around the test field, but found that neither dots nor rings could be painted onto a surface or in to a ring milled into glass or metal in such a way as to give uniform size, and thus uniform brightness. Such nonuniformity would defeat the purpose of an even fixation annulus.

To obtain even distribution of the compound, it must be inserted into a transparent enclosure; the size of the tube's nozzle just fitted into the $1.02-\mathrm{mm}$ inner diameter of plastic (medical) tubing ("silastic" No. 602-201, .040 in. i.d., .085 in. o.d., Dow Corning. Midland, Michigan). The tubing can be evenly filled by gently squeezing out the viscous compound. When the desired length of tubing is completely filled, it is bent into a ring with the two open ends fitted against one another.

A 3-mm-thick piece of aluminum (painted that black) is used to embed the annulus. The circular size of the test tield is cut out of the middle of the plate. Surrounding this opening, a groove is milled to fit the outer diameter of the luminous annulus tube, i.e., about $2 \mathrm{~mm}$ wide and deep. The annulus is wedged snugly into this groove. with its ends fitted together without glue. For added protection. a thin piece of transparent Mylar is placed over the aluminum plate and glued to its corners. The aluminum plate holding the ring and the Mylar cover sheet is mounted onto the faceplate of the centering rotating mount and thus places the annulus in permanent position with respect to the test tield. The complete assembly is shown 


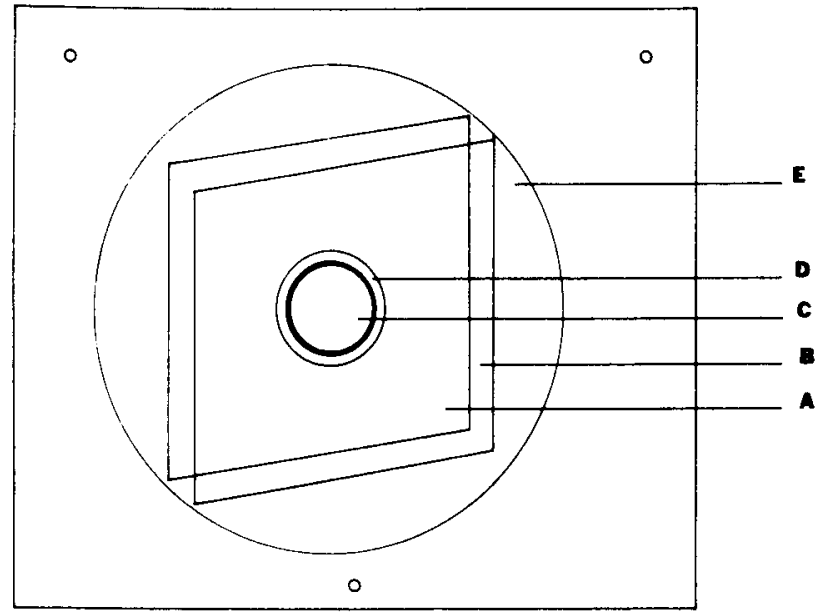

Figure 1. Schematic illustration of test field and annulus. A-Mylar sheet, B-3-mm-thick aluminum plate, C-test field, D-annulus embedded in milled groove, E-centering rotatingmount faceplate.

schematically in Figure 1. An added advantage of this system is the possibility of preparing test field annulus configurations of differing sizes on readily interchangeable aluminum plates.

Annulus brightness was measured with a Spectra Brightness Spot Meter (Model UB 11/2) by removing the artiticial pupil from the viewing tube and aligning the spot meter with its iris diaphragm enclosing the tube opening. The stimulus field (inside the ring) was blocked completely from light. These measurements yielded a ring luminance of $2.7 \times 10^{-3} \mathrm{~mL}$, well within the scotopic range, and our dark-adapted subjects have encountered no problems in locating and tixating its center. Brightness can, of course, be further attenuated by inserting neutral density filters or a continuously adjustable subject-controlled wedge in the pathway, or by using a gelatin neutral density filter in place of the Mylar sheet cover. Such a filter can also be cut into an annulus shape so as to attenuate only the ring brightness and not that of the stimulus tield.

As the luminous compound is completely enclosed in the tube. it does not dry out or separate into granules. Thus, its appearance remains unchanged over time. To ascertain that the same is true for the ring's brightness, measurements with the Spectra Brightness Spot Meter were repeated 6 months after the initial measurement was made. The second set of measurements yielded the same scotopic value as the initial measurement.

Since the compound is referred to as "Yellow," we decided to check its qualitative appearance in the ring by testing 12 color-normal subjects. They were asked to describe the color. if any, of the ring. Six of the subjects described the appearance as achromatic (graly, no color, off-white). The other six described it as having a bluish-white hue. These judgments are in good agreement with responses generally to be expected in the description of scotopic stimuli.

\section{REFERENCES}

Boynton. R. M. Vision. In J. B. Sidowski (Ed.), Experimental merhods and instrumentation in psychology. New York: McGraw-Hill, 1966.

Cornsweet, T. N. Visual perception. New York: Academic Press. 1970.

RigGs. L. Light as a stimulus for vision. In C. H. Graham (Ed.), Vision and visual perception. New York: Wiley. 1965.

(Received for publication December 13, 1974; revision received March $8,1975$. 